\title{
Negative hopping magnetoresistance of two-dimensional electron gas in a smooth random potential
}

\author{
M. E. Raikh, ${ }^{(1)}$ L. I. Glazman ${ }^{(2)}$ \\ (1) Physics Department, University of Utah, Salt Lake City, Utah 84112 \\ (2) Theoretical Physics Institute and Department of Physics, University of Minnesota, \\ Minneapolis, Minnesota 55455
}

\begin{abstract}
We show that the tunnel coupling between semiclassical states localized in different minima of a smooth random potential increases when magnetic field is applied. This increase originates from the difference in gauge factors which electron wave functions belonging to different electron "lakes" acquire in the presence of the field. We illustrate the increase of coupling by a model calculation of tunneling through a saddle point separating two adjacent lakes. In the common case, when the barrier between two lakes is much narrower than their size, the characteristic magnetic field is determined by the area of the lakes, and thus may be quite small. The effect of the field on coupling constants leads to a negative magnetoresistance in low-temperature conduction.
\end{abstract}

PACS Numbers: 73.50.Jt,71.55.Jv 
Deep in the insulating regime, the low-temperature conductivity of a two-dimensional electron gas is dominated by phonon-assisted electron hops between localized states. The conventional picture [目] of hopping implies that localized states are formed by individual impurities. The lower is the temperature, the larger is the separation of the localized states between which a typical hop takes place. In this picture, the magnetic field affects hopping transport in two ways: by shrinking the wave functions of the localized states, and by changing the contributions of different tunneling paths to the amplitude of a hop. The latter means that in the course of tunneling between initial and final states an electron can "visit" a sequence of virtual states localized on neighboring impurities, so that the amplitude of a hop represents a sum of partial amplitudes. In a magnetic field, each path acquires a phase factor. These factors suppress the destructive interference of different paths, thus causing the decrease [2,3] of the resistance in a weak magnetic field. It was shown in [2] that this decrease comes from specific hops for which tunneling amplitudes are close to zero in the absence of a field. Although the portion of these hops is small, the rise of conductivity with magnetic field is related to the increase of the tunneling probabilities for these particular hops, since they are most sensitive to the field.

A different realization of the insulating regime emerges if the random potential is smooth. In the latter case, the electron gas breaks up into separate lakes, each lake accommodating many electrons. Within a certain temperature range the electron transport is provided by tunneling of electrons through saddle points of barriers separating the adjacent lakes. This picture is different from the standard picture of the variable-range-hopping [1], so that the straightforward application of the theory of magnetoresistance [2,3] is impossible. In the present paper, we study magnetoresistance of electron gas in this regime.

It is important to note that the states active in transport (i.e., with energies close to the Fermi level) correspond to high-number levels of size quantization in the minima of the random potential. As a result, the amplitude of a hop is determined not only by the transmission coefficient at the saddle point, but also depends significantly on the overlap between the wave functions on the both sides of the barrier. This overlap is normally small because of the oscillatory behavior of the wave functions. If the applied magnetic field is weak enough, its prime role is to affect this overlap. We argue that the overlap increases with magnetic field, i. e., the coupling between the states, localized in adjacent minima of the potential, becomes stronger. In other words, unlike the conventional [2,3] model, magnetoresistance of each elementary link appears to be negative thus leading to the net negative magnetoresistance of the system.

Consider two minima separated by a barrier. In the vicinity of a saddle point the barrier potential has the form:

$$
U(x, y)=U_{0}-\frac{m \omega_{x}^{2} x^{2}}{2}+\frac{m \omega_{y}^{2} y^{2}}{2},
$$

see Fig. 1. We define the coupling constant between the states $p$ and $k$ in the neighboring minima as a matrix element $t_{p k}$ in the tunneling Hamiltonian:

$$
H=\sum_{p} E_{p}^{l} a_{p}^{\dagger} a_{p}+\sum_{k} E_{k}^{r} b_{k}^{\dagger} b_{k}+\sum_{p k}\left(t_{p k} a_{p}^{\dagger} b_{k}+t_{p k}^{*} b_{k}^{\dagger} a_{p}\right),
$$

where $E_{p}^{l}, E_{k}^{r}$ are the energy levels in the two minima, and $a_{p}^{\dagger}, b_{k}^{\dagger}$ are the corresponding creation operators. The expression for $t_{p k}$ in terms of the wave functions $\psi_{p}^{l}(x, y)$ and $\psi_{k}^{r}(x, y)$ 
can be obtained by generalizing to the two-dimensional case of the procedure employed in the derivation of the tight-binding approximation [4]:

$$
t_{p k}=\frac{\hbar^{2}}{2 m} \iint_{-\infty}^{\infty} d y_{1} d y_{2} G_{E}\left(y_{1}, y_{2}\right) \frac{\partial}{\partial x} \psi_{p}^{l}\left(-\frac{d}{2}, y_{1}\right)\left[\frac{\partial}{\partial x} \psi_{k}^{l}\left(\frac{d}{2}, y_{2}\right)\right]^{\star} .
$$

Here $m$ is the electron mass, and $G_{E}\left(y_{1}, y_{2}\right)$ is the Green function describing the propagation of an electron under the barrier between the points $\left(-d / 2, y_{1}\right)$ and $\left(d / 2, y_{2}\right)$, see Fig. 1 . In the derivation of (3) we have neglected the curvature of the lines of turning points, $U(x, y)=E$, on the both sides of the barrier. The form of the barrier (1) enables one to separate the variables in the region $|x|<d / 2$. As a result the Green function can be expressed in terms of eigenfunctions $\varphi_{n}(y)$ of the harmonic oscillator with frequency $\omega_{y}$,

$$
G_{E}\left(y_{1}, y_{2}\right)=\sum_{n} \frac{\varphi_{n}\left(y_{1}\right) \varphi_{n}\left(y_{2}\right)}{u_{n}^{\prime}(d / 2) v_{n}^{\prime}(-d / 2)-v_{n}^{\prime}(d / 2) u_{n}^{\prime}(-d / 2)},
$$

where $u_{n}(x)$ and $v_{n}(x)$ of the one-dimensional Schroedinger equation with the barrier potential $U_{0}-m \omega_{x}^{2} x^{2} / 2$ and energy $E-(n+1 / 2) \hbar \omega_{y}$. If the transmission coefficient of the barrier is small, one can use the quasiclassical expressions for the functions in (国), $u_{n}, v_{n} \propto \exp \left\{ \pm \int_{0}^{x} d x^{\prime} \kappa_{n}\left(x^{\prime}\right)\right\} / \sqrt{\kappa_{n}}$ with

$$
\kappa_{n}=\frac{\sqrt{2 m}}{\hbar}\left[U_{0}-\frac{m \omega_{x}^{2} x^{2}}{2}-E+\hbar \omega_{y}\left(n+\frac{1}{2}\right)\right]^{1 / 2} .
$$

The precise form of the potential confining the electrons in each lake is a complicated functional of the distribution of positive charges outside the plane of $2 D$ electron system. If the size of a lake exceeds the effective Bohr radius, $a_{B}=\hbar^{2} \varepsilon / m e^{2}$, screening by the electrons of the lake renormalizes considerably the bare confining potential (here $\varepsilon$ is the dielectric constant). However, for a two-dimensional electron gas, the screened potential has still the spatial scale of variations equal to the lake size rather than being equal to a small screening radius [5]. Therefore, we use the simplest parabolic form for the confinement potentials in order to carry out the calculations to the end. We assume

$$
\begin{aligned}
& V_{l}(x, y)=\frac{m \Omega_{l x}^{2}\left(x+D_{1}+d / 2\right)^{2}}{2}+\frac{m \Omega_{l y}^{2} y^{2}}{2}, \\
& V_{r}(x, y)=\frac{m \Omega_{r x}^{2}\left(x-D_{2}-d / 2\right)^{2}}{2}+\frac{m \Omega_{r y}^{2} y^{2}}{2}
\end{aligned}
$$

for the potentials forming the left and right lakes respectively. The corresponding equipotential lines are elliptic with excentricity depending on the corresponding ratios $\Omega_{(l, r) x} / \Omega_{(l, r) y}$. Then the eigenfunctions in each lake are the products of the harmonic oscillator wave functions in $x$ and $y$-directions. Denote with $Y_{l}$ and $Y_{r}$ the turning points of the wave functions in the $y$-direction for the states $p$ and $k$ respectively (see Figure 1). If $Y_{l}$ and $Y_{r}$ exceed significantly the effective size of the tunneling region, the $y$-dependence of $\psi_{p}^{l}\left(-d / 2, y_{1}\right)$ and $\psi_{k}^{r}\left(d / 2, y_{2}\right)$ takes the simple form:

$$
\begin{aligned}
& \psi_{p}^{l}\left(-\frac{d}{2}, y_{1}\right) \propto \sin \left(\frac{m}{\hbar} \Omega_{l y} Y_{l} y_{1}+\chi_{l}\right), \\
& \psi_{k}^{r}\left(\frac{d}{2}, y_{2}\right) \propto \sin \left(\frac{m}{\hbar} \Omega_{r y} Y_{r} y_{2}+\chi_{r}\right),
\end{aligned}
$$


where the phases of $\chi_{l}$ and $\chi_{r}$ are 0 or $\pi / 2$ depending on the parity of the numbers of the harmonic oscillator wave function. Obviously, such a simple form applies when both numbers are high.

As an example, we discuss the coupling between two even (in the $y$-direction) states $\chi_{l}=\chi_{r}=\pi / 2$. Then Eq. (3) reduces to the evaluation of the following double integral:

$$
\begin{aligned}
t_{p k} & \propto \int d y_{1} \int d y_{2} \cos \left(\frac{m}{\hbar} \Omega_{l y} Y_{l} y_{1}\right) \cos \left(\frac{m}{\hbar} \Omega_{r y} Y_{r} y_{2}\right) \\
& \times \sum_{n} \varphi_{n}\left(y_{1}\right) \varphi_{n}\left(y_{2}\right) \exp \left(-\int_{-d / 2}^{d / 2} d x^{\prime} \kappa_{n}\left(x^{\prime}\right)\right) .
\end{aligned}
$$

Note that the integrals over $y_{1}$ and $y_{2}$ can be considered as the Fourier transforms of the functions $\varphi_{n}$. Since for a harmonic oscillator the Fourier transform of the eigenfunction has the same functional form as $\varphi_{n}$, we have

$$
t_{p k} \propto \sum_{n} \varphi_{2 n}\left(Y_{l} \frac{\Omega_{l y}}{\omega_{y}}\right) \varphi_{2 n}\left(Y_{r} \frac{\Omega_{r y}}{\omega_{y}}\right) \exp \left(-\int_{-d / 2}^{d / 2} d x^{\prime} \kappa_{2 n}\left(x^{\prime}\right)\right)
$$

The summation over $n$ can be carried out if we assume the term $\left(n+\frac{1}{2}\right) \hbar \omega_{y}$ in Eq. (5) to be a small correction and expand $\kappa_{n}$ with respect to this term. Then we have

$$
\int_{-d / 2}^{d / 2} d x^{\prime} \kappa_{n}\left(x^{\prime}\right) \approx \frac{\pi\left(U_{0}-E\right)}{\hbar \omega_{x}}+\pi\left(n+\frac{1}{2}\right) \frac{\omega_{y}}{\omega_{x}}
$$

Now we can make use of the addition rule [6] for the functions $\varphi_{n}$ to obtain:

$$
t_{p k} \propto \exp \left[-\frac{\pi\left(U_{0}-E\right)}{\hbar \omega_{x}}-\frac{m\left(Y_{l}^{2} \Omega_{l y}^{2}+Y_{r}^{2} \Omega_{r y}^{2}\right)}{2 \hbar \omega_{y} \tanh \left(\pi \omega_{y} / \omega_{x}\right)}\right] \cosh \left(\frac{m Y_{l} Y_{r} \Omega_{l y} \Omega_{r y}}{\hbar \omega_{y} \sinh \left(\pi \omega_{y} / \omega_{x}\right)}\right) .
$$

The first term in the exponent describes the transmission amplitude for the barrier at the central crossection $y=0$. The other terms describe the reduction of $t_{k p}$ due to the oscillations of the wave functions $\psi_{p}$ and $\psi_{k}$. The expansion of $\kappa_{n}$ (and, hence, Eq(11)) is justified if the main contribution to $\ln t_{k p}$ comes from the tunneling exponent.

The magnetic field $B$ affects the coupling matrix element $t_{k p}$ by modifying the wave functions $\psi_{p}$ and $\psi_{k}$ in the lakes and by changing the Green function Eq. (田) for the motion under the barrier. However, if we choose the guage in the form

$$
A_{x}=0, A_{y}=B x
$$

then the Green function will not be affected (if the width of the tunneling region is small compared to the lake's size). Therefore, we have to find the variation with magnetic field of the wave functions $\psi_{p}, \psi_{k}$ only. Let us start from the left lake centered at $x=-d / 2-D_{1}$. It is convenient first to find the function $\tilde{\psi}_{p}(x, y)$ in the gauge $A_{x}=0, A_{y}=B\left(x+d / 2+D_{1}\right)$ and then to make a transformation to the initial guage Eq.(12). As a result of this transformation, the wavefunction $\psi_{p}$ acquires a gauge phase factor:

$$
\psi_{p}(x, y)=\tilde{\psi}_{p}(x, y) \exp \left[\frac{2 \pi i B}{\Phi_{0}}\left(\frac{d}{2}+D_{1}\right) y\right]
$$


$\Phi_{0}$ being the flux quantum. A similar consideration is applicable to the wave functions in the right lake, and those acquire an additional factor $\exp \left[-2 \pi i B y\left(d / 2+D_{2}\right) / \Phi_{0}\right]$.

It is convenient to write the Schroedinger equation for the function $\tilde{\psi}_{p}$ using the variable $x_{1}=x+D_{1}+\frac{d}{2}$ instead of $x$. Then we have

$$
-\frac{\hbar^{2}}{2 m}\left(\frac{\partial^{2} \tilde{\psi}_{p}}{\partial x_{1}^{2}}+\frac{\partial^{2} \tilde{\psi}_{p}}{\partial y^{2}}\right)-i \hbar \omega_{c} x_{1} \frac{\partial \tilde{\psi}_{p}}{\partial y}+\frac{m}{2}\left[\left(\Omega_{l x}^{2}+\omega_{c}^{2}\right) x_{1}^{2}+\Omega_{l y}^{2} y^{2}\right] \tilde{\psi}_{p}=E \tilde{\psi}_{p}
$$

where $\omega_{c}=e B / \mathrm{cm}$ is the cyclotron frequency. We search for the quasiclassical solution of Eq. (14) in the form $\exp [i S(x, y)]$, where $S$ is the action. For weak enough magnetic fields, $\omega_{c} \ll \Omega_{l x}, \Omega_{l y}$ we can present $S(x, y)$ as $S_{0}+S_{1}$, where $S_{0}$ is the action for motion in the potential $m\left(\Omega_{l x}^{2} x^{2}+\Omega_{l y}^{2} y^{2}\right) / 2$ defined by the equations:

$$
\frac{\partial S_{0}}{\partial x_{1}}=\frac{m \Omega_{l x}}{\hbar} \sqrt{D_{1}^{2}-x_{1}^{2}}, \frac{\partial S_{0}}{\partial y}=\frac{m \Omega_{l y}}{\hbar} \sqrt{Y_{l}^{2}-y^{2}} .
$$

Assuming $S_{1} \ll S_{0}$ we get the following equation for the correction $S_{1}(x, y)$ :

$$
\frac{\hbar^{2}}{m}\left(\frac{\partial S_{0}}{\partial x_{1}} \frac{\partial S_{1}}{\partial x_{1}}+\frac{\partial S_{0}}{\partial y} \frac{\partial S_{1}}{\partial y}\right)=-m \omega_{c} \Omega_{l y} x_{1} \sqrt{Y_{l}^{2}-y^{2}}
$$

It is easy to check that the solution of this equation has the form

$$
S_{1}\left(x_{1}, y\right)=\frac{-m \omega_{c} \Omega_{l y}}{\hbar\left(\Omega_{l y}^{2}-\Omega_{l x}^{2}\right)}\left[\Omega_{l y} x_{1} y+\Omega_{l x} \sqrt{D_{1}^{2}-x_{1}^{2}} \sqrt{Y_{l}^{2}-y^{2}}\right] .
$$

Eq. (17) gives the phase which a state $p$ acquires in the magnetic field. To calculate the coupling coefficient with the help of Eqs. (3) and(13), we need the value of $S_{1}$ at $x_{1}=D_{1}$. It is convenient to present the phase factors [originating from (13) and (17)] for the wave functions $\psi_{p}\left(-d / 2, y_{1}\right)$ and $\psi_{k}\left(d / 2, y_{2}\right)$ as $\exp \left(2 \pi i y_{1} L_{1} / \Phi_{0}\right)$ and $\exp \left(-2 \pi i y_{2} L_{2} / \Phi_{0}\right)$, where

$$
L_{1,2}=\frac{d}{2}+\frac{\Omega_{(l, r) x}^{2}}{\Omega_{(l, r) x}^{2}-\Omega_{(l, r) y}^{2}} D_{1,2} .
$$

After introducing these factors into Eq (3), the calculation of the coupling coefficient is quite similar to the case of zero magnetic field, and yields:

$$
t_{k p}(B)=t_{k p}(0) \exp \left(-\frac{B^{2}}{2 B_{0}^{2}}\right) \cosh \frac{B}{B_{1}} .
$$

It is convenient to express the parameters $B_{0}$ and $B_{1}$ in terms of $L_{1}, L_{2}$, and two amplitudes of zero-point motion, $\lambda_{s}=\sqrt{\hbar / m \omega_{y}}$ and $\lambda_{l, r}=\sqrt{\hbar / m \Omega_{(l, r) x}}$, that characterize respectively the saddle point of the barrier and the lakes,

$$
\begin{aligned}
& B_{0}=\frac{\Phi_{0}}{\sqrt{2} \pi \lambda_{s} \sqrt{L_{1}^{2}+L_{2}^{2}}}\left[\frac{1}{\tanh \left(\pi \omega_{y} / \omega_{x}\right)}+\frac{2 L_{1} L_{2}}{\left(L_{1}^{2}+L_{2}^{2}\right) \sinh \left(\pi \omega_{y} / \omega_{x}\right)}\right]^{-1 / 2} \\
& B_{1}=\frac{\Phi_{0}}{2 \pi L_{1} L_{2}}\left[\left(\frac{\lambda_{s}}{\lambda_{l}}\right)^{2} \frac{Y_{l}}{L_{1}}\left(1+\frac{L_{1}}{L_{2}} \cosh \frac{\pi \omega_{y}}{\omega_{x}}\right)-\left(\frac{\lambda_{r}}{\lambda_{s}}\right)^{2} \frac{Y_{r}}{L_{2}}\left(1+\frac{L_{2}}{L_{1}} \cosh \frac{\pi \omega_{y}}{\omega_{x}}\right)\right]^{-1} \sinh \frac{\pi \omega_{y}}{\omega_{x}} .
\end{aligned}
$$


Correspondingly the magnetoresistance between two lakes behaves as

$$
\frac{R(B)}{R(0)}=\left(\frac{t_{k p}(B)}{t_{k p}(0)}\right)^{-2}=\exp \left(\frac{B^{2}}{B_{0}^{2}}\right) \frac{1}{\cosh ^{2} B / B_{1}} .
$$

The magnetoresistance at small $B$ is quadratic,

$$
\frac{\delta R(B)}{R(0)}=\left(\frac{1}{B_{0}^{2}}-\frac{1}{B_{1}^{2}}\right) B^{2}
$$

and it is negative if $B_{0}>B_{1}$. To estimate the ratio $B_{0} / B_{1}$, we notice that typically all the dimensionless factors in (20) are of the order of unity, and therefore $B_{0} / B_{1} \sim L_{1,2} / \lambda_{s}$. The latter ratio can be estimated as the number of occupied levels in each of the lakes, and is large in the semiclassical regime. Hence, in the small field region the resistance decreases as $\delta R(B) / R(0) \simeq-B^{2} / B_{1}^{2}$, the characteristic value $B_{1}$ being of the order of $\Phi_{0} / D_{1} D_{2}$. The resistance falls off exponentially with $B$ in the region $B_{c} \gg B \gg B_{1}$, where the crossover field is $B_{c}=B_{0}^{2} / B_{1}$. After reaching a minimum at $B \approx B_{c}$, the resistance rises sharply at higher fields. At $B \gg B_{c}$ the exponential increase of the resistance persists. The origin of magnetoresistance in this limit can be understood in terms of the edge states formation. Indeed, it can be easily seen that under the condition $B \gg B_{c}$ the cyclotron radius becomes smaller than the size of the lake. Two edge states on the opposite sides of the barrier correspond to the orbits skipping in opposite directions. Then the mismatch of the wave vectors characterizing these orbits becomes only bigger as the magnetic field increases. Note however that $\mathrm{Eq}(21)$ is not valid at $B \gg B_{c}$ since at $B=B_{c}$ the cyclotron frequency $\omega_{c}$ becomes of the order of $\Omega_{x}$

The physical meaning of the negative magnetoresistance is that the phase factors acquired by the wavefunctions in magnetic field compensate effectively the difference in their wave numbers in the $y$-direction and, therefore, lead to the increase of the coupling. This is clearly seen from the Eq. (3). Indeed, the Green function $G_{E}\left(y_{1}, y_{2}\right)$ connects most effectively points with $y_{1}=y_{2}$, and $G_{E}(y, y)$ is a slow function of its argument. On the other hand, functions $\psi_{k}^{l}(d / 2, y)$ and $\psi_{p}^{r}(d / 2, y)$ rapidly oscillate with different periods. We have shown explicitly on a simple model, that a weak magnetic field reduces the "distance" between the Fourier harmonics belonging to these two wavefunctions thus increasing the overlap between $\partial \psi_{l} / \partial x$ and $\partial \psi_{r} / \partial x$ at the barrier. A similar phenomenon for the "vertical" tunneling between parallel two-dimensional electron systems was considered recently by Lian Zheng and MacDonald [7].

The general case of lakes of an arbitrary shape does not allow one to separe the variables, which may lead to "quantum chaos" 8 10. It might be argued [11 then, that the states $\psi_{l}$ and $\psi_{r}$ are well represented at each point by a random superposition of plane waves travelling in all directions. This superposition, along with other harmonics, would contain the plane wave striking the inter-lake barrier at right angle. Due to the latter harmonic, the coupling matrix elements $t_{k, p}$ would loose the exponential sensitivity to the magnetic field. However the results of the recent studies (see the book by Gutzwiller [8], and Heller's chapter in [12] and references therein) suggest that even in the case of chaotic motion, a substantial portion of quantum eigenstates with high numbers are associated with the periodic classical trajectories in the lake. Namely, these states can be conceived as the result of the BohrSommerfeld quantization of plane waves propagating along these trajectories. The numbers 
of states of the both types described above are of the same order [13]. It means that strong negative magnetoresistance and exponential suppression of conductivity persists for random shapes of the electronic lakes, because of the existence of "scarred" [8, 12] eigenstates.

The magnetoresistance (21) has no temperature dependence. This is because while deriving our main result (20), (21) we assumed the motion of electrons within each lake to be completely coherent. At a finite temperature, the phase-breaking length, $L_{\phi}$, may become smaller than $D_{1,2}$. This will eliminate effectively, the parts of the phase factors (18) acquired due to the electron motion within each of the lakes, i.e. the terms in $L_{1,2}$ proportional to $D_{1,2}$. Therefore at $L_{\phi} \lesssim D_{1,2} L_{1,2}$ in (18) should be replaced by the value of $d / 2$. As a result, in the range of temperatures where $L_{\phi} \sim D_{1,2}$, the magnetoresistance must exhibit a strong temperature dependence, and become weaker at higher temperatures. The sign of the magnetoresistance remains negative, because for a semiclassical electron motion $d$ exceeds $\lambda_{l, r, s}$, and the condition $B_{0} \gg B_{1}$ remains valid, even if $L_{\phi} \lesssim D_{1,2}$.

In conclusion we have demonstrated that the hopping conductivity of two-dimensional electron gas subjected to a smooth random potential should increase drastically with increasing magnetic field. The larger are the electron lakes (the closer is the Fermi level to the classical percolation threshold), the sharper is the raise of the conductivity with magnetic field. The giant negative hopping magnetoresistamce in weak magnetic fields that may be attributed to the mechanism we considered here, was recently reported for GaAs gated heterostructures [14, 15]. However, the observed [14, 15] transition to the metallic (Quantum Hall) state at higher fields can not be accounted for within this simple model.

Discussions with I. L. Aleiner, B. L. Altshuler and H. U. Baranger are gratefully acknowledged. The work at the University of Minnesota was supported by NSF Grant DMR9423244 . 


\section{REFERENCES}

[1] B. I. Shklovskii and A. L. Efros, Electronic Properties of Doped Semiconductors (Springer, Berlin, 1984).

[2] V. L. Nguen, B. Z. Spivak, and B. I. Shklovskii, Pis'ma Zh. Eksp. Teor. Fiz. 41, 35 (1985)[JETP Lett. 41, 42 (1985)]; Zh. Eksp. Teor. Phys. 89, 1170 (1985) [Sov. Phys. JETP 62, 1021 (1985)].

[3] U. Sivan, O. Entin-Wohlman, and Y. Imry, Phys. Rev. Lett. 60, 1566 (1988); O. EntinWohlman, Y. Imry, and U. Sivan, Phys. Rev. B 40, 8342 (1989).

[4] L. D. Landau and E. M. Lifshitz, Quantum Mechanics (Pergamon, New York, 1977).

[5] L. I. Glazman, I. A. Larkin, Semicond. Sci. Tech., 6, 32 (1991).

[6] Higher Transcendental Functions, edited by A. Erdélyi (McGraw-Hill, New York, 1953), Vol. 2.

[7] Lian Zheng and A. H. MacDonald, Phys. Rev. B 47, 10619 (1993).

[8] M. C. Gutzwiller, Chaos in classical and quantum mechanics (Springer-Verlag, New York, 1991).

[9] R. A. Jalabert, A. D. Stone, Y. Alhassid, Phys. Rev. Lett. 68, 3468 (1992);

[10] A. M. Chang, H. U. Baranger, L. N. Pfeiffer, and K. W. West, Phys. Rev. Lett. 73, $2111(1994)$.

[11] M. V. Berry, J. Phys. A 10, 2083 (1977).

[12] Les Houches, Session LII, 1989, Chaos and Quantum Physics, edited by M.-J. Giannoni, A. Voros, and J. Zinn-Justin (North Holland, Amsterdam, 1991).

[13] H. U. Baranger, private communication, November 1994.

[14] H. W. Jiang, C. E. Johnson, and K. L. Wang, Phys. Rev. B 46, 12830 (1992); H. W. Jiang, C. E. Johnson, K. L. Wang, and S. T. Hannahs, Phys. Rev. Lett. 71, 1439 (1993).

[15] T. Wang, K. P. Clark, G. F. Spencer, A. M. Mack, and W. P. Kirk, Phys. Rev. Lett. 72, 1439 (1994). 


\section{FIGURES}

FIG. 1. Two electronic lakes separated by a saddle point $(a)$, and the schematic potential profile in the crossection $\mathrm{y}=0(b)$ 